\title{
Accuracy analysis and application of extrapolation of force-free fields in solar active and quiet regions
}

\author{
Suo Liu ${ }^{1}$, Hongqi Zhang ${ }^{1}$, Jiangtao $\mathrm{Su}^{1}$, and Mutao Song ${ }^{2}$ \\ ${ }^{1}$ Key Laboratory of Solar Activity, National Astronomical Observatories, \\ Chinese Academy of Sciences \\ email: lius@nao.cas.cn \\ ${ }^{2}$ Mountain Observatory, Chinese Academy of Sciences, Nanjing, China
}

\begin{abstract}
In this paper, the availability, applicability and deviation of nonlinear force-free (NLFF) fields extrapolated by Approximate Vertical Integration (AVI), Boundary Integral Equation (BIE) and Optimization (Opt.) methods are studied based on the comparison with two semi-analytical fields (Low \& Lou 1990). These NLFF extrapolations based on the observational vector magnetograms are used to study the spatial magnetic field in the quiet Sun.
\end{abstract}

Keywords. Magnetic Field, Corona, Field Extrapolation.

\section{Introduction}

So far the reliable measurements of the magnetic field are restricted to the photosphere, and the understanding of the magnetic field in the chromosphere and in the corona is a difficult problem due to both intrinsic physical difficulties and observational limitations. Instead of the direct measurement, the chromospheric and coronal magnetic field can be extrapolated from the photospheric magnetic field by assuming the magnetic field above photosphere is force-free (Aly 89). The force-free magnetic field satisfies the follow equations: $\nabla \times \mathbf{B}=\alpha(\mathbf{r}) \mathbf{B}, \nabla \cdot \mathbf{B}=0$. They imply that there is no Lorentz force and $\alpha$ is constant along magnetic field lines $(\mathbf{B} \cdot \nabla \alpha=0$, it means $\alpha$ should be a constant along field lines). If $\alpha=0$, the equations represent a potential field (a current-free field). If $\alpha=$ constant, they describe a current-carrying linear force-free (LFF) field, and if $\alpha=$ $f(r)$ it is a general force-free (NLFF) field. Since the field extrapolation is an important technique to study the corona field, the performance and calculated accuracy of each extrapolation method should be checked specially, also the extrapolation should be applied to observations such as active region and quiet sun.

\section{Extrapolation methods and semi-analytical fields}

Approximate vertical integration (AVI) method Improved from the vertical integration (Song et al., 2006). Assuming magnetic field components is given by

$$
B_{x}=\xi_{1}(x, y, z) F_{1}(x, y, z), B_{y}=\xi_{2}(x, y, z) F_{2}(x, y, z), B_{z}=\xi_{3}(x, y, z) F_{3}(x, y, z) .
$$

Then use approximate vertical integration to calculate field. There exist singular point problems inherently, we do a local integration to avoid it. Here we refer AVI (Song et al. 2006) as original AVI and improved AVI. 
Boundary integral equation (BIE) method Proposed by Yan and Sakurai (2000) using the integration function to extrapolate the field. Parameter (the function of position $\mathrm{x}$ ), must be found through iteration. The integral

$\mathbf{B}\left(x_{i}, y_{i}, z_{i}\right)=\int_{\Gamma} z_{i}[\lambda r \sin (\lambda r)+\cos (\lambda r)] \mathbf{B}_{0}(x, y, 0) / 2 \pi\left[\left(x-x_{i}\right)^{2}+\left(y-y_{i}\right)^{2}+z_{i}^{2}\right]^{3 / 2} d x d y$,

is used, $B_{0}$ is the photosphere magnetic field. Then through iteration to make sure the field is force and divergence-free (cf. He \& Wang, 2008, Li et al. 2004).

Optimization (Opt.) method Developed by Wiegelmann (2004) by minimizing a joint measure for the normalized Lorentz force and the divergence of the field, given by the function,

$$
L=\int_{V} \omega(x, y, z)\left[B^{-2}|(\nabla \times \mathbf{B} \times \mathbf{B})|^{2}+|\nabla \cdot \mathbf{B}|^{2}\right] d^{3} x,
$$

$\omega$ is a weighting function. It minimize $\mathrm{L}$ by optimizing the solution function $\mathrm{B}(\mathrm{x}, \mathrm{t})$ to make it force-free.

Two classic semi-analytical NLFF fields (SAF, the parameter refers paper of Low \& Lou 1990) are used: $\mathrm{SAF} 1: \mathrm{n}=1, \mathrm{~m}=1, \mathrm{l}=0.3, \phi=\pi / 4$, set $\mathrm{x} \in[-0.5,0.5], \mathrm{y} \in[-0.5,0.5]$ and $\mathrm{z} \in[0,1]$ in the Cartesian coordinate system. SAF2: $\mathrm{n}=3, \mathrm{~m}=1, \mathrm{l}=0.3, \phi=4 \pi / 5$, set $\mathrm{x} \in[-0.5,0.5], \mathrm{y} \in[-0.5,0.5]$ and $\mathrm{z} \in[0,1]$. The mesh is $64 \times 64 \times 64$.

\section{Results and Conclusions}

The correlation coefficients between the first semi-analytical field (SAF1) and extrapolated field by BIE and AVI are greater than $90 \%$ in the low solar atmosphere. While for the second semi-analytical field (SAF2), the correlation coefficients are greater than $80 \%$. Although the differences between the semi-analytical solutions and the extrapolated fields exist for both BIE and AVI methods, these two methods can give reliable results for the height of about $15 \%$ of the extent of the lower boundary. The mean values of relative standard deviations (RSD) of a along field lines have been estimated for NLFF field extrapolated by AVI, BIE and Opt. Methods. For SAF1, $<|R S D|>$ of along some selected field lines are 0.96 1.19, 0.63 1.07 and 0.43 0.72 for AVI, BIE and Opt. field, for SAF2, They are 0.80 1.02, 0.67 1.34 and 0.33 0.55 for AVI, BIE and Opt. field, respectively. These numerical NLFF extrapolations based on the Hinode observational vector magnetograms are used to study the spatial magnetic field in the quiet Sun. It is found that the strength differences between the extrapolated NLFF and potential fields exist in the low layers. These differences tend to disappear as the height increases. The analysis shows that the reconstructed NLFF fields deviate significantly from the potential field and the azimuths are different among three NLFF fields.

\section{References}

Aly, J. J. 1989, Sol.Phys., 120, 19

He, H. \& Wang, H. 2008, Jgr, 113, A05S90

Li, Z, Yan, Y. H., \& Song, G. 2004, MNRAS, 347, 1255

Liu, S., Zhang, H. Q., Su, J. T., \& Song, M. T. 2011, Sol.Phys., 269, 41

Liu, S., Zhang, H. Q., \& Su, J. T. 2011, Sol.Phys., 270, 89

Liu, S., Zhang, H. Q., \& Su, J. T. 2012, Ap\&SS, 337, 665

Low, B. C. \& Lou, Y. Q. 1990, Apj, 352, 343

Song, M. T., Fang, C., Tang, Y. H., Wu, S. T., \& Zhang, Y. A. 2006, Apj, 649, 1084

Wiegelmann, T. 2004, Sol.Phys., 219, 87

Yan, Y. \& Sakurai, T. 2000, Sol.Phys., 195, 89 